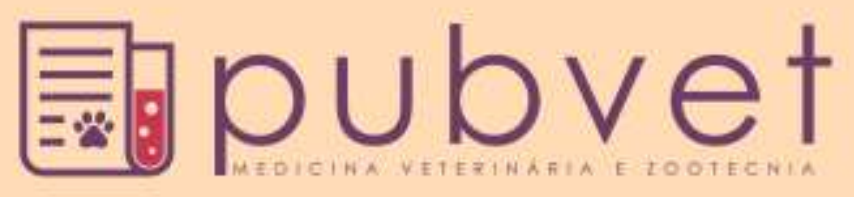

HTTP://DX.DOI.ORG/10.22256/PUBVET.V11N3.217-223

\title{
Influência da temperatura ambiente no verão na produção de leite de vacas holandesas
}

\author{
Sheila Tavares Nascimento ${ }^{1 *}$, Yasmin Piovan Rossetto ${ }^{2}$, Alessandra Aparecida Silva ${ }^{3}$, \\ Priscilla Ayleen Bustos Mac-Lean ${ }^{4}$, Juliana Paula Lozada Tenório ${ }^{2}$ \\ ${ }^{1}$ Professora da Universidade de Brasília, Faculdade de Agronomia e Medicina Veterinária, Brasília-DF, Brasil. E-mail: sheila.tn@ gmail.com \\ ${ }^{2}$ Zootecnista, autônoma, Maringá-PR, Brasil.E-mail: sc_yasmin@hotmail.com; julianaplozada@gmail.com \\ ${ }^{3}$ Professora da Universidade Estadual de Maringá, Departamento de Zootecnia, Maringá-PR, Brasil. E-mail: teczoo@hotmail.com \\ ${ }^{4}$ Professora da Universidade Estadual Paulista "Julio de Mesquita Filho", Departamento de Engenharia de Biossistemas, Tupã-SP, Brasil. \\ E-mail: priscilla.abm@gmail.com \\ *Autor para correspondência
}

\begin{abstract}
RESUMO. O trabalho teve como objetivo avaliar a perda na produção de leite diretamente influenciada pelo clima sem a utilização de qualquer recurso para aquecimento e/ou resfriamento das instalações numa propriedade leiteira. Foram utilizadas 20 vacas da raça Holandesa com peso médio de $480 \mathrm{~kg}$, em diferentes estágios de lactação. Foi mensurada a produção de leite, para cada animal, e a temperatura ambiente durante a ordenha, no período de trinta dias, duas vezes ao dia, nos manejos de ordenhas realizados pela manhã e à tarde. Para tal, formaram-se três grupos de animais, sendo: GRUPO L - vacas em diferentes estágios de lactação; GRUPO PL - vacas no pico de lactação; GRUPO P - vacas em primeira lactação. Os dados da produção de leite foram submetidos à análise de variância, considerando os efeitos dos dias de observação, dos grupos de animais em cada um dos períodos (manhã e tarde) e considerando a temperatura do ar uma covariável do modelo. Estimou-se a correlação de Pearson $(\mathrm{P}<0,05)$ entre a temperatura do ar e a produção de leite dos animais, assim como a transferência de calor por mecanismos sensíveis e latentes das vacas em relação ao ambiente para os dois períodos analisados. Houve diferença significativa $(\mathrm{P}<0,05)$ para a produção de leite entre os períodos de ordenha para todos os grupos avaliados. Há uma correlação negativa e significativa $(\mathrm{P}<0,05)$ da temperatura do ar sobre a produção, mensurado em $\mathrm{Kg}$ de leite. A ineficiência da dissipação de calor devido a um aumento da temperatura do ar torna o período vespertino crítico para a ordenha dos animais. Portanto, torna-se necessário a utilização de sistemas de climatização para diminuir o impacto negativo da temperatura do ar sobre o desempenho de vacas leiteiras.
\end{abstract}

Palavras chave: Bem-estar animal, estresse térmico, produção de leite

\section{Influence of air temperature during the summer on milk yield of Holstein cows}

ABSTRACT. The aim of this work was to evaluate milk yield losses directly influenced by the climate without the use of installments' resources to heating and/ or cooling in a dairy farm. Twenty Holstein cows with an average body weight of $480 \mathrm{~kg}$ and different lactation stages were used. The milk yield to each animal and the air temperature during milking were measured for 30 days, twice a day, in the morning and in the evening milking. The cows were divided in three groups, as follows: GROUP L - cows in different lactation periods; GROUP PL - cows in the peak of lactation; GROUP P - cows in the first lactation. Data of milk production were submitted to an analysis of variance, considering the effects of days of observation, of the groups of the animals in each period (morning and afternoon) and considering the air temperature as a covariable of the model. The Pearson correlation 
was estimated $(\mathrm{P}<0.05)$ between the air temperature and the milk yield, as well as the heat transfer by sensible and latent flows between the cows and the environment to both periods evaluated. There was a significant difference $(\mathrm{P}<0.05)$ for milk yield among the morning and afternoon milking to all the groups studied. There is a negative and significant correlation $(\mathrm{P}<0.0001)$ of the air temperature on milk yield, measured as $\mathrm{kg}$ of milk. The inefficiency of heat dissipation due to an increase of air temperature makes the afternoon period critical to the milking of cows. Therefore it is necessary to use acclimatization systems to decrease the negative impact of air temperature on the performance of dairy cows.

Keywords: Animal welfare, thermal stress, milk yield

\section{Influencia de la temperatura ambiente en verano sobre la producción de leche en vacas Holstein}

RESUMEN. El objetivo del estudio fue evaluar la pérdida en la producción de leche directamente influenciada por el clima sin el uso de ningún recurso para la calefacción y/o refrigeración de las instalaciones en una propiedad lechera. Fueron utilizadas 20 vacas Holstein con un peso promedio de $480 \mathrm{~kg}$, en diferentes etapas de lactancia. Para cada animal se midió la producción de leche, y la temperatura ambiente durante el ordeño, en treinta días, dos veces al día, en los ordeños realizados por la mañana y por la tarde. Con este fin, se formaron tres grupos de animales: GRUPO L - vacas en diferentes etapas de lactación; GRUPO PL - vacas en el pico de lactación; GRUPO P - vacas en primera lactancia. Los datos de producción de leche se sometieron a análisis de varianza, teniendo en cuenta los efectos de los días de observación, los grupos de animales en cada uno de los periodos (mañana y tarde) y teniendo en cuenta la temperatura del aire como una covariable en el modelo. Se estimó la correlación de Pearson $(\mathrm{P}<0,05)$ entre la temperatura del aire y la producción de leche, así como la transferencia de calor por los mecanismos sensibles y latentes de las vacas en relación con el medio ambiente por los dos periodos analizados. Hubo una diferencia significativa $(\mathrm{P}<0,05)$ para la producción de leche entre los periodos de ordeño para todos los grupos evaluados. Existe una correlación negativa y significativa $(\mathrm{P}<0,05)$ de la temperatura del aire en la producción, medida en $\mathrm{kg}$ de leche. La ineficiencia de la disipación de calor debido a un aumento en la temperatura del aire es un periodo crítico para el ordeño de la tarde de los animales. Consecuentemente, se hace necesario el uso de sistemas de refrigeración para reducir el impacto negativo de la temperatura del aire sobre el rendimiento de vacas lecheras.

Palabras clave: Bienestar animal, estrés por calor, producción de leche

\section{Introdução}

A produção de leite ocupa lugar de destaque entre os produtos de origem animal, não só pelo valor comercial, mas também pela importância de seu consumo. A produção brasileira de leite cresceu entre os anos de 2011 a 2014 a uma taxa média anual de $3,1 \%$; aumentando em volume de 32,0 bilhões de litros para 35,1 bilhões de litros produzidos (ANUALPEC, 2015). O Brasil, além de ser um grande produtor de leite, também é um grande importador e suas produções são voltadas principalmente para atender a um mercado interno de grandes proporções.

A raça de gado holandês destaca-se por animais de grande porte, alto peso, grande consumo de matéria seca (MS), e alta produção leiteira, possuem boa habilidade materna, úbere grande, e ligamentos fortes para sustentação do grande volume de leite, são dependentes de temperatura amena (Passetti et al., 2016).

O conforto térmico é alcançado quando as vacas leiteiras são expostas a um ambiente em que seja possível a manutenção de sua taxa basal sem que seja preciso dispor de suas reservas corpóreas e/ou ativação de mecanismos fisiológicos para manutenção de sua temperatura corporal (Silva, 2000, Pinheiro and Brito, 2009), ou seja, sua zona termoneutra é delimitada por uma temperatura critica superior e inferior, e esse é um ponto de bastante relevância, pois, os nutrientes contidos na dieta dos animais serão utilizados visando um maior desempenho produtivo e de crescimento 
(Baêta and Souza, 2010). Maia et al. (2005) observaram que acima de $30^{\circ} \mathrm{C}$, as perdas de calor sensível de vacas holandesas é praticamente nulo; enquanto que a evaporação cutânea, representada pela sudação, corresponde a até $85 \%$ de toda a dissipação de calor latente. Esses resultados indicam a ineficiência dos animais manterem sua temperatura corporal, comprometendo consequentemente a produção de leite da raça Holandês em ambiente tropical.

O objetivo deste trabalho foi avaliar o impacto da temperatura do ar na produção de leite e na transferência de calor de vacas da raça holandesa quando mantidas em sistemas de criação sem o uso de climatização durante a estação do verão no estado do Paraná.

\section{Material e Métodos}

$\mathrm{O}$ experimento foi conduzido numa fazenda pertencente ao município de Paiçandu, no estado do Paraná, a cerca de 30 quilômetros da cidade de Maringá.

A propriedade está localizada na latitude $23^{\circ}$ 27' 27" S; longitude $52^{\circ} 02^{\prime} 55^{\prime \prime} \mathrm{W}$ e 470 metros de altitude; segundo a classificação de Köppen and Geiger (1928), o clima é classificado como Cfa (clima subtropical, caracterizado por temperatura média no mês mais frio inferior a $18^{\circ} \mathrm{C}$ e temperatura média no mês mais quente acima de $22^{\circ} \mathrm{C}$, com verões quentes, geadas pouco frequentes e tendência de concentração das chuvas nos meses de verão, contudo sem estação seca definida). No município de Paiçandu a temperatura média anual é de $18.9{ }^{\circ} \mathrm{C}$, e a pluviosidade de 1306 milímetros.

Foram utilizadas 20 vacas holandesas, com peso médio de $480 \mathrm{~kg}$, divididas em diferentes estágios de lactação: Grupo L - composto por vacas em lactação, fora do pico; Grupo PL composto por vacas no pico de lactação; Grupo P - composto por vacas primíparas, ou seja, em primeira lactação. As vacas eram mantidas a pasto, sendo arrebanhadas diariamente para a ordenha, duas vezes ao dia (às 09:00h e às 15:30h) e eram alimentandas com ração concentrada nos mesmos períodos dos dias. Durante todo o experimento os animais receberam água, pasto de coast-croos e sal mineral ad libitum, enquanto o fornecimento de ração concentrada era restrito, sendo ofertada duas vezes ao dia após a ordenha, em instalações cobertas. $\mathrm{O}$ alimento era disponibilizado por cerca de trinta minutos.
Avaliou-se na sala de ordenha as produções diárias de leite $(\mathrm{kg})$, sendo quantificadas as produções na ordenha no período da manhã, no período da tarde e a produção total diária, caracterizada pelo somatório entre os dois períodos.

A coleta de dados foi realizada durante trinta dias, entre os meses de dezembro de 2013 e janeiro de 2014, por meio de anotações diárias da produção dos grupos citados acima. De acordo com o registro numérico de cada animal, a produção de leite foi individualizada e estimada emergindo-se uma régua graduada dentro dos baldes, desprezando-se a espuma.

Para a avaliação do ambiente térmico, realizaram-se medições da temperatura do ar em cada ordenha, durante os 30 dias de observação, por meio de um termômetro instalado próximo à entrada dos animais na sala de ordenha, permanecendo durante todo o período de coleta. As mensurações de produção leiteira e temperatura do ar foram diárias, nos períodos matutino e vespertino, tendo-se a produção de leite por vaca/dia considerando-se a soma das duas ordenhas.

Estimou-se a transferência de calor sensível e latente (W. $\mathrm{m}^{-2}$ ) dos animais em função da temperatura do ar, utilizando-se as equações propostas por Maia et al. (2005) para vacas Holandesas em clima tropical, descritas a seguir:

$$
\begin{gathered}
G=237,33-7,35 \cdot t_{a r}-0,0022 \cdot t_{a r}^{2} \\
E=85,18 \cdot e^{\left(\frac{t_{a r}-24,92}{7,96}\right)}
\end{gathered}
$$

onde:

$\mathrm{G}=$ transferência de calor sensível por convecção e radiação de ondas longas das vacas holandesas, W. $\mathrm{m}^{-2}$

$\mathrm{t}_{\mathrm{ar}}=$ temperatura do $\mathrm{ar},{ }^{\circ} \mathrm{C}$;

$E=$ transferência de calor latente por evaporação cutânea e evaporação respiratória das vacas holandesas, $\mathrm{Wm}^{-2}$.

Os dados da produção de leite foram analisados estatisticamente com auxílio do programa Statistical Analysis System (SAS, versão 9.2) de acordo com Littell et al. (1998). O programa foi utilizado para organização dos arquivos, exame de distribuição dos dados com relação à normalidade, estatísticas de tendência central, de dispersão, de associação e análise de variância, sendo esta baseada no método dos quadrados mínimos (Harvey, 1960). As médias para cada um dos 
fatores englobados no modelo foram analisadas pelo teste de Tukey, considerando-se um nível de $5 \%$ de significância. A análise de variância foi realizada utilizando o seguinte modelo estatístico, considerando a temperatura do ar uma covariável:

onde:

$$
Y_{i j k l m}=a+D_{i}+G_{K}\left(P_{l}\right)+b_{1} T+e
$$

$\mathrm{Y}_{\mathrm{ijk} k \mathrm{~m}}$ é a m-ésima observação da produção de leite; a é o intercepto; D é o efeito fixo do i-ésimo dia de observação (i=1, ..., 30); G é o efeito fixo do k-ésimo grupo de vacas $(\mathrm{k}=$ grupo $\mathrm{L}, \mathrm{P}, \mathrm{PL})$ dentro do l-ésimo período de coleta $(1=$ manhã ou tarde); bé o coeficiente de regressão linear sobre a temperatura do ar; e é o erro aleatório.

Foi realizada uma análise de correlação de Pearson entre a temperatura do ar e produção de leite, utilizando-se o procedimento procCORR da ferramenta SAS a fim de se procurar uma relação entre esses dados.

Por fim, foram elaborados modelos de análise de regressão linear, utilizando-se o procedimento procREG do SAS, para a predição da produção de leite de acordo com a variação da temperatura do ar e para vacas em diferentes estágios de lactação.

\section{Resultados e Discussão}

Considerando o quadro da análise de variância, observaram-se diferenças para a produção de leite $(\mathrm{P}<0,05)$ entre as temperaturas do ar observadas $\mathrm{e}$ entre os grupos de vacas dentro dos períodos de avaliação (Tabela 1); enquanto que não houve diferença significativa $(\mathrm{P}>0,05)$ entre os 30 dias de experimento.

Tabela 1. Quadrados médios da produção de leite observados durante o experimento

\begin{tabular}{lcc}
\hline Fonte de Variação & GL & QM \\
\hline Dias & 29 & $0,17^{\mathrm{NS}}$ \\
Grupos (Período) & 5 & $1596,67^{*}$ \\
Temperatura & 1 & $6,17^{*}$ \\
Resíduo & 1164 & 3,58 \\
\hline $\mathrm{R}^{2}$ & - & 0,80 \\
\hline $\mathrm{CV}$ & - & 17,76 \\
\hline Total & 1199 &
\end{tabular}

$\mathrm{O}$ efeito dos dias não foi significativo ( $\mathrm{P}>0,05)$; porém, durante o período de observação notou-se uma variação muito grande de temperatura do ar, cujo efeito foi significativo ( $\mathrm{P}<0,05)$, o que pode ser explicado pela estação do ano. Os meses de dezembro e janeiro, período em que o experimento foi conduzido, são caracterizados pela ocorrência da estação verão no hemisfério sul, marcado por altas temperaturas, aumento expressivo nas precipitações e dias mais longos (Figura 1). Ao traçarmos uma linha na figura 1 indicando de acordo com Maia et al. (2005) a temperatura na qual a transferência de calor sensível passa a ser nula e a evaporação torna-se a principal via de dissipação de calor, observam-se vários dias em que a temperatura média observada foi superior a esse valor.

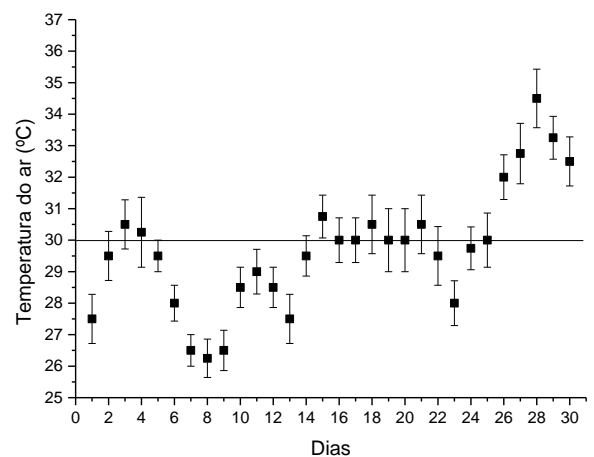

Figura 1. Médias da temperatura do ar observadas ao longo dos 30 dias de experimento na sala de ordenha numa fazenda da região de Maringá, $P R$.

No entanto, ao observar-se a temperatura do ar durante os períodos matutino e vespertino avaliados na pesquisa, nota-se que a mesma foi superior aos $30^{\circ} \mathrm{C}$ para todos os dias avaliados durante o período da tarde (Figura 2).

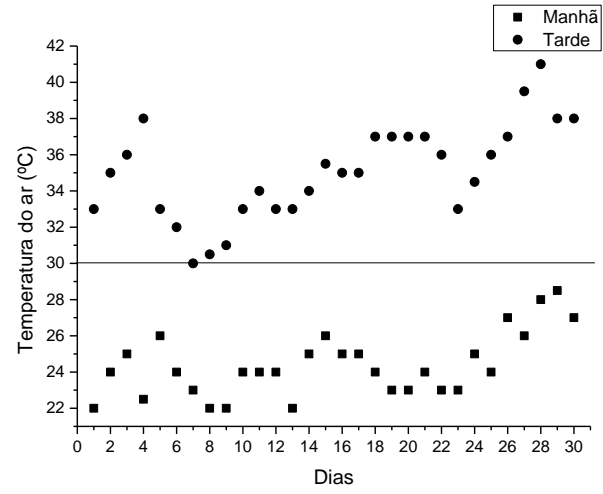

Figura 2. Médias da temperatura do ar observadas nos períodos da manhã e da tarde ao longo dos 30 dias de experimento na sala de ordenha numa fazenda da região de Maringá, PR.

Observou-se consequentemente uma grande variação da temperatura do ar dentro da sala de ordenha entre os períodos da manhã e da tarde (Figura 2), sendo em alguns dias superior a $10^{\circ} \mathrm{C}$. Yano et al. (2014) corroboram o impacto das variáveis ambientais sobre o desempenho, produção e qualidade do leite de vacas Holandesas durante os meses do verão. O mesmo foi 
verificado nessa pesquisa, onde ao compararem-se os dados da produção entre os períodos da manhã e da tarde, observou-se uma diminuição na quantidade de leite mensurada na ordenha realizada à tarde em comparação com a da manhã $(\mathrm{P}<0,05)$, para os três grupos de animais estudados, primíparas, em lactação ou em pico de lactação (Figura 3). A produção diária de leite observada mostrou-se aquém dos resultados esperados, ou seja, algo em torno de $20 \%$ de perdas relacionando a primeira à segunda ordenha para os três grupos de animais avaliados.

A análise de correlação confirma a influência da temperatura do ar sobre a produção de leite $(r=$ $-0,63, \quad \mathrm{P}<0,0001)$, ou seja, o aumento da temperatura do ar afeta negativamente a produção de leite. Essa correlação negativa e significativa foi observada dentro de cada um dos grupos estudados: para animais em lactação (Grupo L), em pico de lactação (Grupo PL) e primíparas (Grupo P), o coeficiente de correlação observado foi igual a $-0,80,-0,73$ e $-0,90$, respectivamente $(\mathrm{P}<0,0001)$.

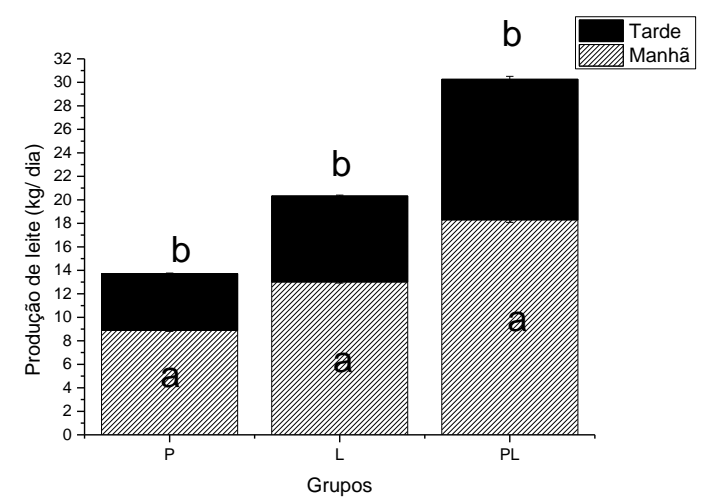

Figura 3. Produção de leite (kg/ dia) de vacas Holandesas durante a ordenha nos períodos da manhã e da tarde, para os grupos: P - vacas primíparas; L - vacas em lactação; PL vacas em pico de lactação. Letras diferentes em cada um dos grupos avaliados indicam diferença significativa em relação à produção de leite entre os períodos da manhã e da tarde pelo teste de Tukey $(\mathrm{P}<0,05)$.

Temperatura elevada, bem como variações climáticas acentuadas, como oscilações na umidade e radiação, não se enquadram como condições adequadas para a criação de vacas para exploração leiteira. E em especial, a raça holandesa não se favorece com tal situação, podendo deixar de expressar seu potencial genotípico (Vasconcellos et al., 2003). A redução na produção de leite é inversamente proporcional ao aumento da temperatura, uma vez que os eventos envolvidos na produção de leite culminam na geração de calor (Berbigier, 1988). Sendo assim, um fator que pode ter influenciado diretamente a diferença produtiva de leite entre os dois períodos de ordenha pode ser a temperatura do ar.

O melhoramento genético na produção de leite é diretamente relacionado ao aumento da ingestão alimentar, e consequentemente o aumento da ingestão resulta em maior produção de calor metabólico. Por fim, o aumento da produção de calor metabólica exige mecanismos termorreguladores eficazes para que as vacas mantenham a sua temperatura corporal e a homeostase fisiológica (Kadzere et al., 2002).

O impacto e influência da temperatura do ar sobre a produção de leite pode ser observado ao estimarmos a transferência de calor por mecanismos sensíveis e latentes dos animais utilizando-se as equações propostas por Maia et al. (2005) propostas a partir da mensuração em vacas holandesas em clima tropical (Figura 4). Sabendose que os mecanismos sensíveis de transferência de calor são aqueles dependentes de um diferencial de temperatura entre a superfície do animal e o ambiente para ocorrer, observa-se que com o aumento da temperatura do ar há uma menor capacidade de dissipação de calor por radiação de ondas longas e convecção, como se pode verificar durante a ordenha realizada no período vespertino (Figura 4b). Os valores negativos da transferência de calor (Figuras $4 \mathrm{a}$ e 4b) representam as temperaturas do ar na qual os animais ganham calor do ambiente, ao invés de dissiparem essa quantidade de energia. Portanto, os animais durante o período matutino estão em melhores condições de conforto, pois dissipam calor para o meio tanto por mecanismos sensíveis quanto latentes, sendo que os mecanismos de transferência de calor latente (via evaporação cutânea e respiratória) aumentam sua importância para manutenção da homeostase dos animais em torno de $24^{\circ} \mathrm{C}$ de temperatura do ar (Figura $4 \mathrm{a}$ ).

Por sua vez, as vacas durante a ordenha da tarde foram expostas a condições de estresse térmico, e a transferência de calor por mecanismos sensíveis tornou-se uma via de ganho de calor, o que agrava o estresse térmico desses animais (Figura 4b). Consequentemente, os animais precisaram utilizar os mecanismos latentes de transferência de calor como vias disponíveis para a dissipação de calor e tentativa de manutenção da temperatura corporal. As perdas latentes, representadas pela sudação e evaporação respiratória são indesejáveis para vacas durante o 
período de lactação, pois indicam uma maior utilização da energia corporal que deveria ser utilizada para a produção de leite (Young, 1975).

A

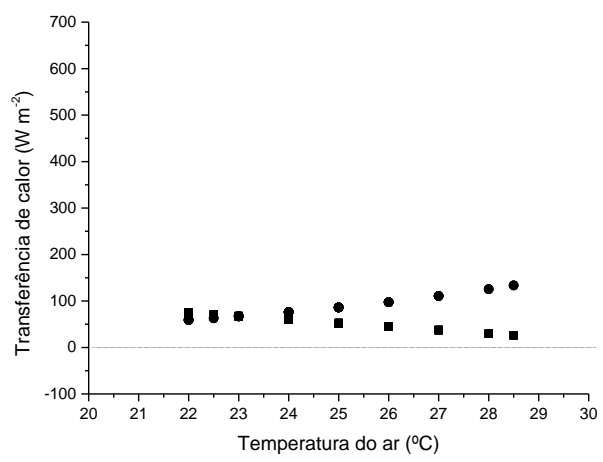

B

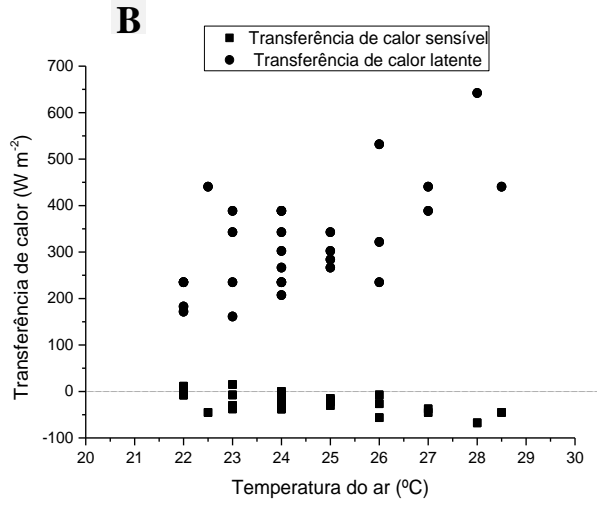

Figura 4. Transferência de calor sensível e latente de vacas holandesas na sala de ordenha em uma propriedade da região de Maringá, PR - A) durante o período da manhã; B) durante o período da tarde.

A produção leiteira no Brasil possui rebanhos com alta heterogeneidade, principalmente os provenientes da raça holandesa são os que possuem maior diferença produtiva, mas isso não se dá só pela genética, mas também pela diferença ambiental. De acordo com Torres et al. (2000) a duração da lactação ocorre mais por fatores ambientais do que genéticos. Os autores ressaltaram que se produtores de baixa e média produção realizassem práticas de manejo e redução de restrições ambientais tais como: controle da temperatura, sombreamento, temperatura da água adequada, consumo de matéria seca em quantidade e qualidade, estado sanitário etc., esses poderiam ter animais com uma expressão completa do seu material genético.

Para cada um dos grupos, considerando o estágio produtivo das vacas holandesas, puderamse determinar equações de regressão linear para a predição da produção de leite $\left(\mathrm{kg} \cdot \mathrm{dia}^{-1}\right)$ de acordo com a temperatura do ar (Tabela 2).

Tabela 2. Equações de regressão linear para a predição da produção diária de leite $(\mathrm{kg})$ para vacas primíparas, em lactação e em pico de lactação em função da temperatura do ar $\left({ }^{\circ} \mathrm{C}\right)$.

\begin{tabular}{lcc}
\hline \multicolumn{1}{c}{ Grupo de vacas } & \multicolumn{1}{c}{ Equação } & $\begin{array}{c}\mathrm{R}^{2} \\
\text { ajustado }\end{array}$ \\
\hline $\begin{array}{l}\text { Primíparas } \\
\text { (Grupo P) }\end{array}$ & Produção de leite $=16,87-0,34 . \mathrm{tar}^{\mathrm{a}}$ & 0,81 \\
$\begin{array}{l}\text { Em lactação } \\
\text { (Grupo L) }\end{array}$ & Produção de leite $=23,99-0,46 . \mathrm{tar}$ & 0,64 \\
$\begin{array}{l}\text { Pico de lactação } \\
\text { (Grupo PL) }\end{array}$ & Produção de leite $=30,52-0,52$. tar & 0,53 \\
\hline
\end{tabular}

Uma série de fatores poderiam ser repensados ou reformulados a fim de incrementar a produção leiteira de vacas holandesas mantidas sob altas temperaturas (estação do verão). Portanto, estratégias para reduzir o estresse térmico devem ser desenvolvidas para permitir que as vacas expressem todo o seu potencial genético na propriedade (Kadzere et al., 2002), como a adoção de sistemas de climatização e disponibilidade de sombreamento no pasto na propriedade.

\section{Conclusão}

A temperatura do ar é uma variável que afeta diretamente a produção de leite de vacas holandesas em qualquer estágio da fase de lactação. A influência negativa da temperatura do ar é mais severa durante o período vespertino, em que os animais desviam parte da energia corporal para a manutenção da temperatura corporal, diminuindo a produção de leite. Estratégias de climatização devem ser utilizadas pelas propriedades a fim de se diminuir o impacto das altas temperaturas observadas durante o verão sobre a produção diária de leite.

\section{Referências}

ANUALPEC. 2015. Anuário da Pecuária Brasileira, 20th edn. Instituto FNP, São Paulo, SP, Brasil.

Baêta, F. C. \& Souza, F. C. 2010. Ambiência em edificações rurais: conforto animal. Universidade Federal de Viçosa, Viçosa.

Berbigier, P. 1988. Bioclimatologie des ruminants domestiques en zone tropicale. INRA, Paris.

Kadzere, C. T., Murphy, M. R., Silanikove, N. \& Maltz, E. 2002. Heat stress in lactating dairy cows: a review. Livestock Production Science, 77, 59-91. 
Köppen, W. \& Geiger, R. 1928. Klimate der Erde. Gotha: Verlag Justus Perthes. Wall-map $150 \mathrm{~cm} \times 200 \mathrm{~cm}$.

Littell, R. C., Henry, P. R. \& Ammerman, C. B. 1998. Statistical analysis of repeated measures data using SAS procedures. Journal of Animal Science, 76, 1216-31.

Maia, A. S. C., Silva, R.G. \& Loureiro, C. M. B. 2005. Sensible and latent heat loss from the body surface of Holstein cows in a tropical environment. International Journal of Biometeorology, 50, 17-22.

Passetti, R. A. C., Eiras, C. E., Gomes, L. C., Santos, J. F. d. \& Prado, I. N. 2016. Intensive dairy farming systems from Holland and Brazil: SWOT analyse comparison. Acta Scientiarum. Animal Sciences, 38, 439-446.

Pinheiro, A. A. \& Brito, F. I. 2009. Bem-estar e produção animal. EMBRAPA, Sobral.

Silva, R. G. 2000. Introdução à bioclimatologia animal. Nobel, São Paulo.

Torres, R. A., Bergmann, J. A. G., Costa, C. N., Pereira, C. S., Valente, J., Penna, V. M., Filho, R. d. A. T. \& Araújo, C. V. 2000. Heterogeneidade de variância e avaliação genética de bovinos da raça Holandesa no
Brasil. Revista Brasileira de Zootecnia, 29, 1050-1059.

Vasconcellos, B. F., Pádua, J. T., Muñoz, M. F. C. \& Tonhati, H. 2003. Efeitos genéticos e ambientais sobre a produção de leite, o intervalo de partos e a duração da lactação em um rebanho leiteiro com animais mestiços, no Brasil. Revista Universidade Rural, 23, 39-45.

Yano, M., Shimadzu, H. \& Endo, T. 2014. Modelling temperature effects on milk production: a study on Holstein cows at a Japanese farm. SpringerPlus, 3, 129-140.

Young, B. A. 1975. Some physiological costs of cold climates. Missouri Agricultural Experiment Station. Agricultural Experiment Station, University of Missouri-Columbia, Missouri.

\section{Article History:}

Received 8 November 2016

Accepted 19 December 2016

Available on line 18 January 2017

License information: This is an open-access article distributed under the terms of the Creative Commons Attribution License 4.0, which permits unrestricted use, distribution, and reproduction in any medium, provided the original work is properly cited. 